\title{
Zur Provenienz einer Sammlung von Telemann-Kirchenmusiken in der Österreichischen Nationalbibliothek (Mus. Hs. 15.532)
}

\author{
von Ralph-Jürgen Reipsch (Magdeburg)
}

In Heft 3/2008 der Musikforschung stellte Eric Fiedler die im Titel genannte, in Werner Menkes Telemann-Vokalwerke-Verzeichnis ${ }^{1}$ noch nicht berücksichtigte Sammlung von Kirchenmusiken Georg Philipp Telemanns vor, identifizierte die Werke, ordnete sie teilweise den jeweiligen Jahrgängen zu und stellte Überlegungen hinsichtlich der Provenienz des Materials an. ${ }^{2}$ Wohl aufgrund des Vorhandenseins einer solchen Sammlung in einer österreichischen Bibliothek sowie durch eine leider ungenaue Papieruntersuchung kam Fiedler zu dem Schluss, dass es sich um Manuskripte österreichisch-ungarischer Herkunft handeln würde. ${ }^{3}$ Eine Annahme, die, wie hier in gebotener Kürze gezeigt werden soll, nicht zu halten ist.

\section{Zum Besitzgang}

Nach Auskunft der Musikabteilung der Österreichischen Nationalbibliothek wurden die 41 Telemann-Abschriften ${ }^{4}$ im Jahre 1842 durch Ankauf aus dem Besitz des bekannten Musikaliensammlers Franz Hauser (1794-1870) erworben. ${ }^{5}$ Etwa um 1838 hatte dieser sich als Gesangslehrer in Wien niedergelassen. ${ }^{6}$ Hauser besaß hier gute Kontakte zu Sammlerkreisen, insbesondere zu Aloys Fuchs, so dass eine Verbindung zur damaligen Hofbibliothek nicht schwer herzustellen gewesen sein dürfte. Warum Hauser die Manuskripte veräußerte, ist nicht bekannt. In seiner Sammlung haben sich später noch einige andere Telemann-Werke befunden, ${ }^{7}$ zum Schwerpunkt haben diese indes nicht gehört. Die 1842 veräußerten Manuskripte hatte Hauser vermutlich erworben, als er sich zwischen 1832 und 1837 in Leipzig und Berlin aufhielt. Man könnte vermuten, dass sie aus der Musikaliensammlung Johann Gottfried Schichts stammen, die 1832 in Leipzig versteigert wurde. Aus Georg Poelchaus Handexemplar des Auktionskataloges geht hervor, dass Hauser hier neben Kompositionen von Johann Sebastian Bach u. a. auch Telemann-Werke ersteigert hat. Zu den angebotenen

${ }^{1}$ Werner Menke, Thematisches Verzeichnis der Vokalwerke von Georg Philipp Telemann, Frankfurt a. M. 1982 f.

${ }^{2}$ Eric Fiedler, „, Telemann in Wien - zur Wiederentdeckung von sieben als verschollen geltenden Kantaten aus Telemanns ,Zweitem Lingen'schen Jahrgang' in der Österreichischen Nationalbibliothek", in: $M f 61$ (2008), S. $205-221$.

${ }^{3}$ Fiedler erwähnt bei seinen Überlegungen, „dass es sicherlich reizvoll“ wäre, "die Entwicklung der evangelischen Kirchenmusik in Sopron/Ödenburg - erhaltene Archivalien vorausgesetzt - bis ins 18. Jahrhundert weiter zu verfolgen". Dass dies in der Tat lohnenswert sein kann, zeigt die aus der evangelisch-lutherischen Gemeinde der Stadt stammende Musikaliensammlung, in der immerhin zwei Telemann-Abschriften zu verzeichnen sind. Vgl. Kornél Bárdos, Sopron zenéje a 16-18. században. A müvek tematikus jegyzékét összeállította Vavrinecz Veronika, Budapest 1984, S. 539 (Katalog Nr. 650 „Bist du denn gar von Stahl und Eisen“ TVWV 20:45 [weltliche Kantate]; Katalog Nr. 651 „Liebe, die vom Himmel stammet" TVWV 1:1044). Ich danke Herrn Prof. András Székely (Budapest) für diesen Hinweis.

4 Im Katalog von Mantuani werden fälschlich 42 Kirchenmusiken angegeben, da im zweiten Band der Sammlung unter Nr. 19 ein notierter Lesungstext für „Vor Mittags" (2 Petri 1,19) als „Textus sine notis musicis" mitgezählt wird. Im Haupteintrag indes steht korrekt „numero 41“. Vgl. Josef Mantuani, Codicum musicorum. Pars 1. Cod. 1550117500 (= Tabulae codicum manu scriptorum praeter Graecos et orientales in Bibliotheca Palatina Vindobonensis asservatorum 9), Wien 1897, S. 8-10, hier: S. 10. Fiedler (S. 205) bringt den genannten Lesungstext unnötigerweise mit TVWV 1:1663 in Verbindung.

5 Schreiben von Dr. Günter Brosche, dem damaligen Direktor der Musiksammlung, an den Verfasser vom 30. Juli 1996.

${ }^{6}$ Zu Franz Hauser vgl. vor allem Yoshitake Kobayashi, Franz Hauser und seine Bach-Handschriftensammlung, Diss. Göttingen 1973.

${ }^{7}$ Einige Telemann zugeschriebene Kantaten gelangten über Karl Anton, der die Reste des Hauser-Archivs verwaltete, im Jahre 1956 an die Hessische Landes- und Hochschulbibliothek Darmstadt. Jedoch nicht alle dieser im TVWV fälschlich als komplett verschollen gekennzeichneten Kantaten stammen von Telemann. Vgl. Ralph-Jürgen Reipsch, „Die anonym überlieferte Kantate ,Willkomm, du Licht aus Licht geboren' - eine Komposition von Telemann oder Fasch?", in: Das Wirken des Anhalt-Zerbster Hofkapellmeisters Johann Friedrich Fasch für auswärtige Hofkapellen. Bericht über die Internationale Wissenschaftliche Konferenz am 20. und 21. April 2001 im Rahmen der 7. Internationalen FaschFesttage in Zerbst (= Fasch-Studien 8), Dessau 2001, S. 161-190, hier: S. 176-181. 
Kirchenmusiken Telemanns gehören eine Markuspassion und „49 Cantaten Part. zusammen 199 Bg. ${ }^{\prime 8}$, die unter einem Los zusammengefasst waren. Allerdings ist Poelchaus handschriftlichem Eintrag zu entnehmen, dass Hauser nur die Markuspassion erworben hat, für die "Cantaten" vermerkt er keinen Käufer. Außerdem irritiert die Anzahl der angebotenen Kantaten, denn sie unterscheidet sich von der in Wien überlieferten Sammlung (49 statt 41 Stücke). Auch die angegebene Bogenzahl (199 Bögen, d. h. 796 Seiten) korreliert nicht mit der realen Anzahl der Blätter (205 Blätter, d. h. 410 Seiten). Somit bleibt vorerst im Dunkeln, aus welcher Quelle Hauser die Manuskripte tatsächlich erworben hat. Allerdings ist es anhand der Papierbefunde und eines weiter unten zu erläuternden Indizes immerhin möglich nachzuweisen, dass die "Wiener" Telemann-Quellen in den mitteldeutschen Raum (zwischen Harz und Thüringen) zu verorten sind.

\section{Papierbefund}

Die meisten der ermittelten Wasserzeichen lassen sich der Papiermühle Ilfeld (bei Nordhausen) zuordnen, ${ }^{9}$ die zwischen 1693 und 1739 durch den Pächter Samuel Walther ${ }^{10}$ und bis 1748 durch dessen Witwe geführt wurde. ${ }^{11}$ Dies betrifft die nachstehenden Formpaare (Hauptmarke/Gegenmarke): schreitender Hirsch / SW; springendes Pferd / SW; Posthorn, darunter SW. Ein weiteres Wasserzeichen (zweihenklige Blumenvase / AHS) stammt aus einer Quedlinburger Papiermühle. Daneben treten weitere Wasserzeichen auf: Pferd / ICE; Tulpen in zweihenkliger Vase / GBI; undeutliche Hauptmarke (gekreuzte Schwerter?)/ keine Gegenmarke.

Eine vergleichbare Papierkonstellation ist Armin Brinzing zufolge in der Sammlung Bösenrode (heute D-Gs) auszumachen, ${ }^{12}$ vor allem in den Abschriften von Johann Georg Nattermann (geb. 1689), der zwischen 1715 und 1744 als Kantor daselbst tätig war: ${ }^{13}$ Hirsch / SW; ${ }^{14}$ springendes Pferd / SW; ${ }^{15}$ Blumenstrauß in Vase / AHS. ${ }^{16}$ Der kleine Ort Bösenrode, am Kyffhäuser in der Goldenen Aue gelegen, ist nur etwa $30 \mathrm{~km}$ entfernt von der ehemals am Harzrand befindlichen Ilfelder Papiermühle. Doch das Verbreitungsgebiet des Ilfelder Papiers ist nicht auf die erweiterte Harzregion beschränkt. Für den Thüringer Raum lässt es sich z. B. in autographen Manuskripten des Sondershäuser Kapellmeisters Johann Balthasar Christian Freislich belegen. ${ }^{17}$

\footnotetext{
${ }^{8}$ Versteigerungs-Katalog der von dem verstorbenen Herrn J. G. Schicht, Cantor an der Thomasschule zu Leipzig hinterlassenen Musikalienhandlung, welche | als Anhang der Bücherauction vom 19ten November [dies gestrichen und ersetzt mit: d. 19. Decbr. 1832] zu Leipzig den Meistbietenden gegen baare Zahlung überlassen werden soll. Leipzig (D-B Ac 931, Nachlass Georg Poelchau), S. 19.

${ }^{9}$ Mitteilungen zur Herkunft der Papiere von Andrea Lothe (Deutsches Buch- und Schriftmuseum der Deutschen Nationalbibliothek Leipzig, Papierhistorische Sammlungen), Wasserzeichenmappe zur Papiermühle Ilfeld Sign. II 419/0/2.

10 Vgl. Johann Sebastian Bach, „Inventionen und Sinfonien“. Kritischer Bericht von Georg von Dadelsen und Klaus Hofmann (= Neue Ausgabe sämtlicher Werke [NBA] V/3), Kassel u. a. 2007, S. 30, Anm. 30.

11 Die Bach-Quellen der Sing-Akademie zu Berlin. Katalog, bearbeitet von Wolfram Enßlin, Bd. 2, Hildesheim u. a. 2006, S. 651 (Wasserzeichen Nr. 36).

12 Armin Brinzing, „Mitteldeutsche Kirchenmusik in der Staats- und Universitätsbibliothek Göttingen“", in: Zwischen Musikwissenschaft und Musikleben. Festschrift für Wolf Hobohm zum 60. Geburtstag am 8. Januar 1998, hrsg. von Brit Reipsch und Carsten Lange (= Magdeburger Telemann-Studien 17), Hildesheim u. a. 2001, S. 203-215. Siehe auch die Daten zu dieser Sammlung in RISM, Serie A/II: Musikhandschriften nach 1600 <http://opac.rism.info/index.php>, 15.10.2010.

13 Brinzing, S. 205 und 207.

14 Z. B. in der 1724 datierten Partiturabschrift von Liebholds „Das ist rechter Glaubens Mut" , D-Gs $8^{\circ}$ Cod. Ms. philos. 84e: Liebhold 5.

15 Z. B. in der auf 1722 datierten Abschrift Nattermanns von „Herr, erhöre meine Stimme ${ }^{\prime \prime}$ TVWV 1:738, D-Gs $8^{\circ}$ Cod. Ms. philos. 84e: Telemann 8 (B.c.-Stimme) sowie in seiner Partiturabschrift von „Die Hoffnung ist mein Leben“ TVWV 20:48, D-Gs $8^{\circ}$ Cod. Ms. philos. 84e: Telemann 4 von 1725.

16 Z. B. in Nattermanns Abschrift (Tenor- und Violinstimme) von „Ich hebe meine Augen auf zu den Bergen“ TVWV 7:15, D-Gs $8^{\circ}$ Cod. Ms. philos. 84e: Telemann 9.

17 Dies betrifft Papiere mit dem Wasserzeichen springendes Pferd / SW. Vgl. Karla Neschke, Johann Balthasar Christian Freislich (1687-1764). Leben, Schaffen und Werküberlieferung. Mit einem Thematisch-systematischen Verzeichnis seiner Werke (= Schriftenreihe zur Mitteldeutschen Musikgeschichte, Serie 2: Forschungsbeiträge 3), Oschersleben 2000, passim, Abb. S. 406.
} 


\section{Formale Abweichungen}

Einige der in Wien überlieferten Kirchenmusiken zeigen eine vom Original abweichende musikalische Form: Ihnen wurde jeweils ein Kirchenliedsatz vorangestellt. Es betrifft z. B. neun der zehn in dieser Sammlung enthaltenen Kompositionen aus dem sogenannten „Jahrgang ohne Recitativ"18 (1724/25) nach Dichtungen von Johann Friedrich Helbig und Benjamin Neukirch. ${ }^{19}$ Diese Eigentümlichkeit ${ }^{20}$ lässt sich außerhalb der Wiener Sammlung bis dato nur in zwei weiteren Quellen zu diesem Jahrgang beobachten. So in einer in Frankfurt a. M. überlieferten, vermutlich aus dem mitteldeutschen Raum stammenden Partitur ${ }^{21}$ und in einem Erfurter Textdruck. ${ }^{22}$ Diesem zufolge führte der Kantor Johann Martin Klöppel (1697-1755) am zweiten Ostertag 1736 die Kirchenmusik zum dritten (!) Ostertag „Ich weiß wohl, was ich für Gedanken“ TVWV 1:880 auf, die hier mit dem Choralsatz „Allein Gott in der Höh" eröffnet wurde. Die Voranstellung eines Kirchenliedes im vierstimmigen Kantionalsatz scheint auf eine regionale Aufführungs- bzw. Verbreitungstradition hinzuweisen, die als weiteres Indiz für die Eingrenzung der Herkunft der in Wien aufbewahrten Manuskripte dienen kann.

Nachgetragen sei, dass in dieser mitteldeutschen Sammlung von ordentlichen Kirchenmusiken Georg Philipp Telemanns eine sonst nicht bekannte Bezeichnung für einen der Telemann-Jahrgänge anzutreffen ist. Bei der Kantate „Aus Gnaden seid ihr selig worden“ TVWV 1:112 (Bd. 1, Nr. 10), die aus dem sogenannten „ersten Lingenschen Jahrgange ${ }^{\prime 23}$ stammt, vermerkte der Schreiber auf der Partitur "themat.[ischer] Jahrg:[ang]".

Die Entstehungszeit der in dieser Sammlung vereinten Werke, die vorrangig aus verschiedenen Kirchenmusik-Jahrgängen Telemanns stammen, liegt zwischen 1710 und $1749 .{ }^{24}$ Eine weiterführende Untersuchung der Manuskripte, die zu konkreteren Ergebnissen in Hinblick auf die Schreiber bzw. die Provenienz und die Zeit der Entstehung führen könnte, steht noch aus.

18 Ralph-Jürgen Reipsch, „Telemanns ,Jahrgang ohne Rezitativ““, in: Telemann und die Kirchenmusik. Bericht über die Internationale Wissenschaftliche Konferenz, Magdeburg, vom 15. bis 17. März 2006 anläßlich der 18. Magdeburger Telemann-Festtage (in Vorbereitung).

${ }^{19}$ Bei „Glaubet nicht einem jeglichen Geist" TVWV 1:627 ist ein Kirchenliedsatz zwar nicht notiert, es wird jedoch ein Textincipit angegeben. Bei „Der Segen des Herrn“ TVWV 1:1751 sind nur Melodie und Text ausgeschrieben. Ohne Choral erscheint lediglich „Der Herr hat gesagt" TVWV 1:260.

${ }^{20}$ Nur zwei Stücke des „Jahrgangs ohne Recitativ“ mit vorangestelltem Choralsatz können in dieser Form als von Telemann autorisiert angesehen werden, da sie auch in den originalen Hamburger Textbüchern so erscheinen: „Ach Gott, wie manches Herzeleid“ TVWV 1:20 und „Herzliebster Jesu, was hast du verbrochen“ TVWV 1:785. Diese befinden sich jedoch nicht in dem Wiener Manuskript.

21 „Der Herr ist mein Hirte" TVWV 1:266, D-F Mus Hs 966 (ehemals Manskopfsches Museum, Sign. 1942:30, vgl. <http://opac.rism.info/search?documentid=455002138>, 15.10.2010). Diese Partitur weist neben dem oben erwähnten Ilfelder Wasserzeichen (Pferd / SW) eine Notationseigentümlichkeit auf, die auch typisch für die Wiener Sammlung ist: Vokalstimmen und Basso continuo sind eine kleine Terz tiefer notiert und orientieren somit auf den Chorton, die Streicherstimmen hingegen stehen in der Originaltonart. Demnach könnte diese Partitur aus dem gleichen Überlieferungsfeld wie die Wiener Manuskripte stammen. Der Schreiber ist dort allerdings nicht nachzuweisen.

22 TEXTE zur Kirchen=MUSIC, welche Auf die heilige Oster=Tage, Jubilate und Himelfahrth=Christi In der Barfüsser =Kirche Im Jahr 1736. sollen musiciret werden, GOtt zu Ehren, Und Zur Ermunterung heiliger Andacht überreicht von Joh. Martin Klöppel. Cant. RFFURTH [d. i. Erfurt], Gedruckt bey Georg Andr. Müllers sel. hinterlassen Wittbe (Exemplar der Bibliothek des Zentrums für Telemann-Pflege und -Forschung Magdeburg).

23 So bezeichnet bei Friedrich Wilhelm Marpurg, Kritische Briefe über die Tonkunst, 4. Teil, Berlin 1760, S. 382. Vgl. auch Brit Reipsch, „Die Telemannquellen in Goldbach - der ,erste Lingensche Jahrgang'“, in: Telemann-Beiträge. Abhandlungen und Berichte, 3. Folge, hrsg. von Wolf Hobohm und Brit Reipsch (= Magdeburger Telemann-Studien 15), Oschersleben 1997, S. 64-95.

${ }^{24}$ Vertreten sind die Jahrgänge „Geistliches Singen und Spielen“ (der früheste, 1710/11 in Eisenach entstandene Jahrgang), der "erste Concertenjahrgang", der "Sicilianische Jahrgang", der erste und zweite „Lingen'sche Jahrgang", der „Jahrgang ohne Recitativ", der Hamburger Jahrgang 1726/27, der als Vorlage für den Druck „Auszug derjenigen Árien ..." (Hamburg 1727) diente, sowie der sogenannte „Engel-Jahrgang". Letzterer erschien 1748/49 im Druck und ist somit der späteste hier vertretene Jahrgang. Neben den Kirchenmusiken aus Jahrgängen ist eine Ode aus Erdmann Neumeisters "Zugang zum Gnaden-Stuhl“ (zuerst Weißenfels 1705) enthalten. Vgl. zu Telemanns Kirchenmusik-Jahrgängen grundsätzlich Wolf Hobohm, „Telemann als Kantatenkomponist - Versuch einer Ordnung und Typologie seiner Jahrgänge", in: „Nun bringt ein polnisch Lied die gantze Welt zum Springen“. Telemann und Andere in der Musiklandschaft Sachsens und Polens, hrsg. von Friedhelm Brusniak (= Arolser Beiträge zur Musikforschung 6), Sinzig 1998, S. 29-52; ders., ", Telemann als Kantatenkomponist zwischen 1710 und 1730", in: Telemann in Frankfurt. Bericht über das Symposium Frankfurt am Main, 26./27. April 1996, hrsg. von Peter Cahn (= Beiträge zur Mittelrheinischen Musikgeschichte 35), Mainz u. a. 2000, S. 55-73. 